\title{
Morphology classification and behaviors identification of birds in scientific video
}

\author{
Can Chen ${ }^{1}$, Yan Xiong and Baoping Yan²
}

\begin{abstract}
Through the establishment of video sensor networks, allowing researchers to do long-term field observations better in their offices. But the study of morphology classification and behavior identification of animals from mass video data is very costly and time-consuming repetitive work. For this, we did video preprocessing and multiple targets recognition and tracking. We built a classification model by support vector machine algorithm. Used 384 morphological records of bar-headed geese from the scientific video obtained in Qinghai Lake, we extracted a vector constructed 9 features in-cluing aspect ratio, shape factor and $7 \mathrm{Hu}$ invariant moments respectively from each record and thus got 384 vectors in total, in which 289 were used for training and 95 for testing. The morphology classification accuracy reached $65.3 \%$. And we used bar-headed geese movement parameters and bar-headed geese posture information; we analyzed and identified barheaded geese four basic behaviors, and achieve a certain recognition rate. This will help the researches to do study the normal behavior and abnormal behavior of the bar-headed geese and the reserve personnel to do daily monitoring of the barheaded geese.
\end{abstract}

Keywords: birds behaviour analysis and identify - target detection and tracking . morphologyclassification - feature vector - support vector machine

\author{
${ }^{1}$ Can Chen $(\square)$ \\ General Group for Advancing e-Science Applications \\ Computer Network Information Center, Chinese Academy of Science \\ Beijing, China \\ e-mail: chencan@cnic.cn \\ This paper is supported by Director Fund of CNIC(CNIC_ZR_201102) \\ ${ }^{2}$ Yan Xiong \\ General Group for Advancing e-Science Applications \\ Computer Network Information Center, Chinese Academy of Science \\ Beijing, China \\ Baoping Yan \\ General Group for Advancing e-Science Applications \\ Computer Network Information Center, Chinese Academy of Science \\ Beijing, China
}




\section{Introduction}

Observation activitiesofanimalsoften need tolast a long time. Alsowe need observation withoutinterferenceas far as possible.Thisgreatly limits thestudy of animal behavior. Through the establishment ofvideosensor networks, allowingresearchersto dofield observationsin their office. Because they do not need to enter the wilderness environment, the researchers can easier tomake long-termstudy. Meanwhile, we do some proper camouflage to the video sensors, as far as possible into its natural environment, so animals can be observed without interference. Researchers can also save the video for later analysis and comparison studies. Through the application of video sensor networks can greatly facilitate the study of animal behavior.

We deployed network video monitoring systemin main area of Qinghai Lake, monitoring birds hatching, breeding, and feeding. Now, we deployed 23 HDvideo monitors (as Fig.1.1), covering all 5 core regions. There are about 100GB video data generatedevery day, get a lot of valuable information hidden.

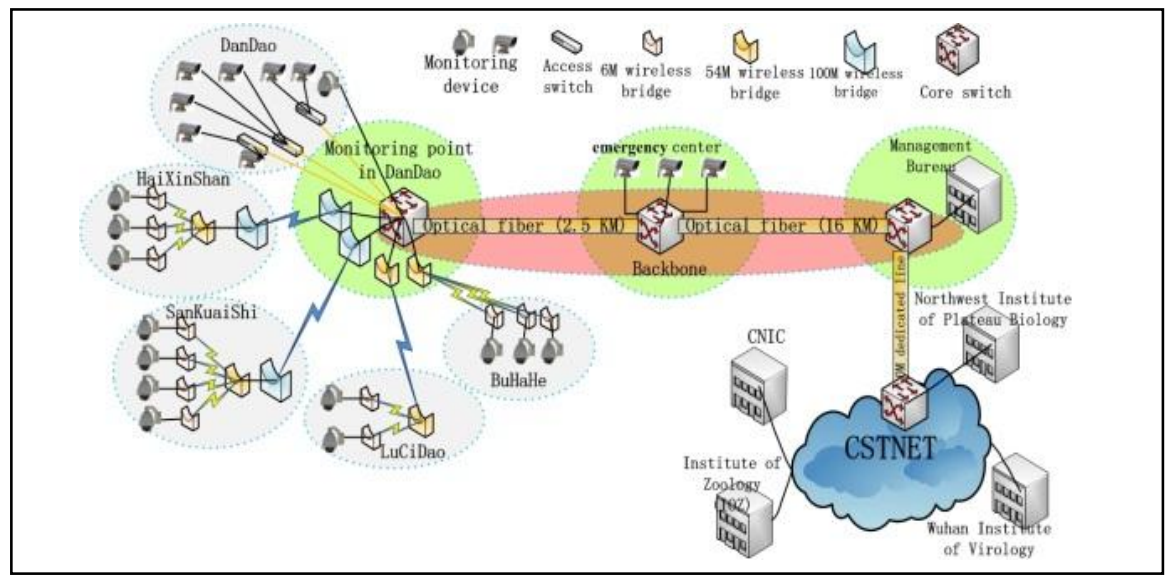

Fig. 1.1Video monitor network of Qinghai Lake

However, such a large number of video, simply rely on researchers to study is a time-consuming things, and will put a lot of energy and time wasted in the repetitive work. For this, we studied the birds' behavior identification techniques in field research video. So that the computer can understand the animal behavior in video, do calibration work to help researchers to conduct common behavior, and save the researchers' time and energy. In addition, through the research of animal common behavior, abnormal behavior can be identification, the computer can give alarm information of abnormal animal behavior, notice the researchers and protected area management office staff.

We need to study the preprocessing of video file, animal behavior model framework, animal target detection, animal target tracking, animal posture classification, feature selection and extraction, motion parameters acquisition, animal beha- 
vior analysis and recognition algorithm. Through these, to achieve a certain recognition rate of the recognition of the animal common behavior in field research video.

At present, domestic and foreign scholars research on video behavior analysis are mainly concentrated in the following two aspects: (1) Movement and behavior analysis of animals such as mice in the fixed independent scene $[1,2,3,4]$, these researches are aim at laboratory fixed small areas, and easy to calibration recognition the target; (2) Aiming at action and mutual interaction of different kinds of animals in fixed scene $[5,6,7,8]$. Through the understanding and analysis of research status at home and abroad, we found that there is no such research.

\section{Target detection and tracking}

Animal target tracking plays a significant role in subsequent links as animal motion parameters extraction, animal behavior analysis, etc. Through the analysis and comparison of all kinds of algorithms, we choose and improve the camshiftalgorithm to do animal target tracking.

In order to realize multi-targets tracking, and solve the problem of target tracking failure, we improve the camshift algorithm. We use binary difference method to update the history information of target motion, with the specified target size threshold and constrained relationship, we can realize multi-targets tracking and when the target tracking failure it will not appear abnormal.

We implemented the algorithm based on the OpenCV library [9]. We analyzed the video of Qinghai Lake, detection and tracking the bar-headed geese target in the video. Using two modes:

Manually click on the target in the video window; program will automatically track the click on the target. The experimental results show as Fig. 2.1:

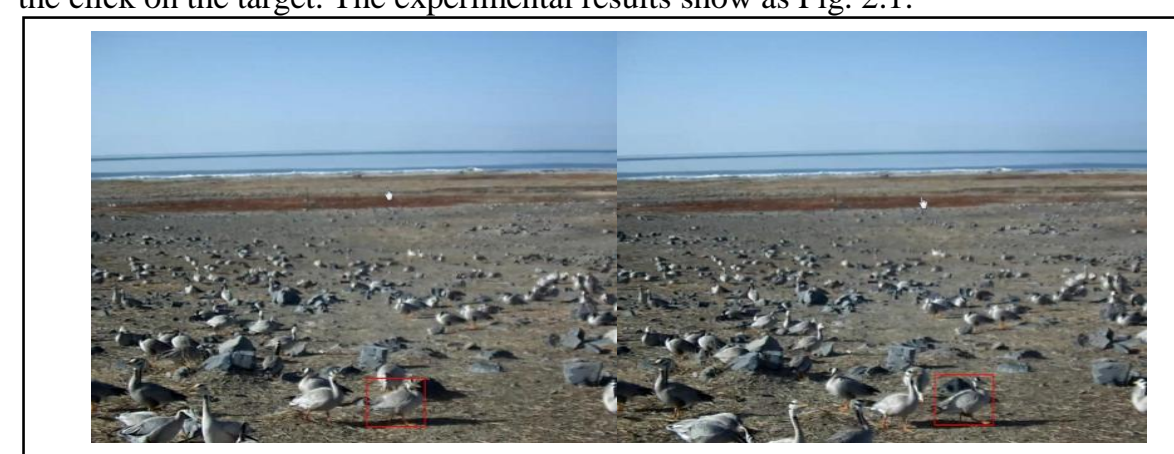

Fig. 2.1Manually click track the target

Input the threshold of the size of the moving target, program can track all moving targets which conform to the threshold value. We improved the Camshift algo- 
rithm, by comparing the difference of adjacent image frames; we got all the search windows which center position and size are changed, the program tracked the windows which size bigger than the threshold value. The experimental results show as Fig. 2.2:

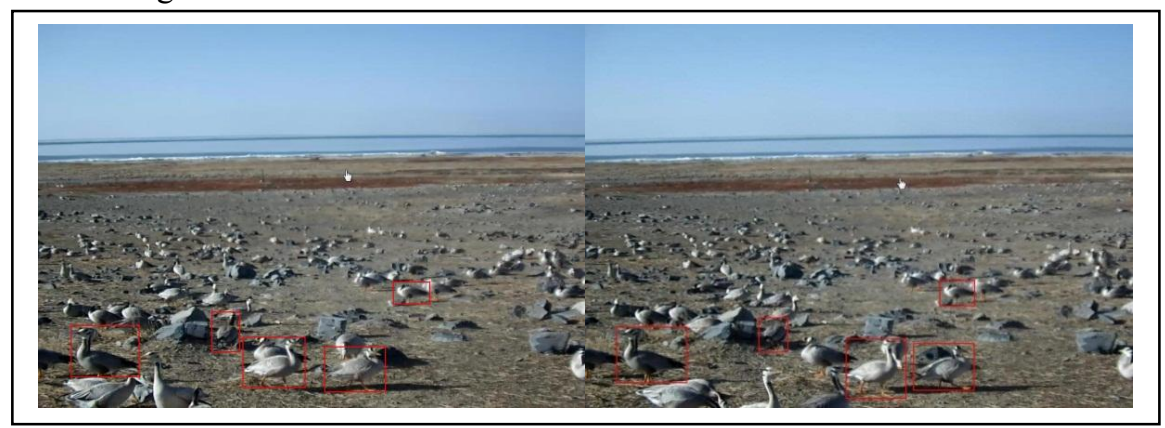

Fig. 2.2Automatic tracking motion object conforms to the threshold

After experiments we proof, moving object detection and tracking based on OpenCV can detection and tracking moving objects on the basis of real-time display image. And the detection and tracking has higher robustness. In the process of tracking, even the target remain static, the program will not lost the target tracking.

\section{Morphology Classification}

In this section, we research the animal target posture classification and recognition technology; research the shape feature extraction technology, to extract effective features from the animals, as input parameters of a posture classifier. Research data classification technology; build training and testing samples for classifier.

\subsection{Morphology Definition}

We defined four kinds of morphologies in total, and they are standing, bending, crouching and winging respectively. The morphologies and their descriptions are shown as Fig.3.1 and Table 3.1. 


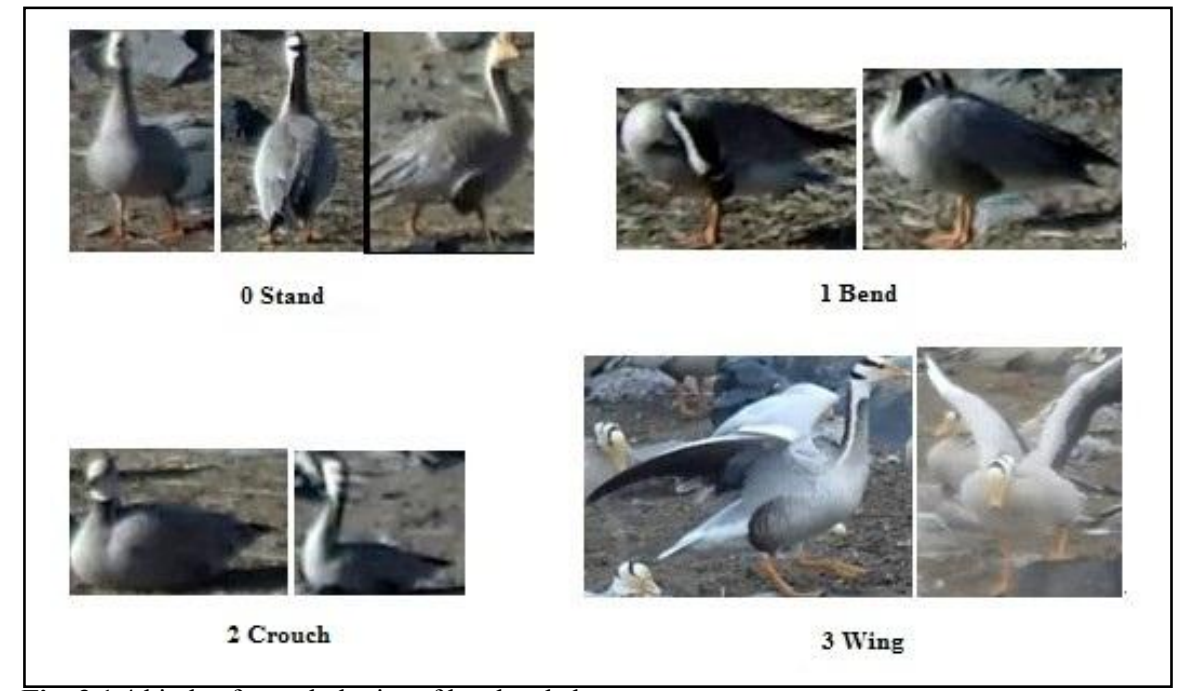

Fig. 3.1 4 kinds of morphologies of bar-headed geese

Table 3.1Morphology Description

\begin{tabular}{lll}
\hline $\mathbf{N}$ & Name & Description \\
\hline $\mathbf{0}$ & standing & Uprighthead, neck and leg \\
$\mathbf{1}$ & bending & Curledneck \\
$\mathbf{2}$ & crouching & Uprighthead and neck, curled leg \\
$\mathbf{3}$ & winging & Opened wing \\
\hline
\end{tabular}

\subsection{Experiment}

Support Vector Machine (SVM) is an excellent supervised learning method for classification problems. It takes a training set composed of a list of records as its input and builds a model as the output. Each record is a feature vector containing a series of attributes and a hand marking label. The process is shown as Fig. 3.2.

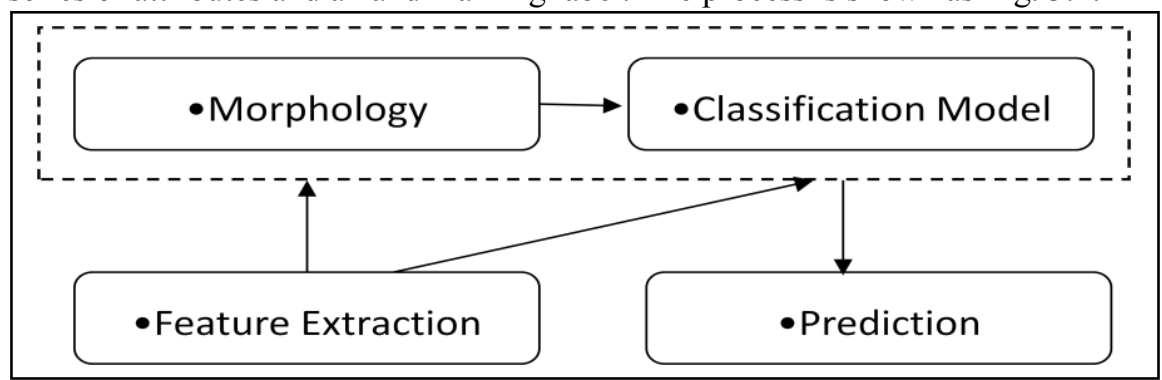

Fig. 3.2SVM work process 


\subsubsection{Feature Extraction}

Feature extraction is very important for model building in SVM. Appropriate features can highly promote the computing efficiency and prediction accuracy. In our experiment, we extract 9 features in total. They are area factor $F$ which defines as the number the target's area divide perimeter, aspect ratio $\mathrm{R}$ representing the number length divide width, and $7 \mathrm{Hu}$ invariant moments.

\subsubsection{Data Sets}

We collect 289 records for training set and 95 records for test set. Their distributions are shown as Table 3.2, in which $0,1,2,3$ are the indexes of 4 morphologies.

Table 3.2The distributions of training set and test set

\begin{tabular}{llllll}
\hline set & total & $\mathbf{0}$ & $\mathbf{1}$ & $\mathbf{2}$ & $\mathbf{3}$ \\
\hline training & 289 & 102 & 67 & 45 & 90 \\
test & 95 & 19 & 24 & 27 & 25 \\
\hline
\end{tabular}

Each record is a 10-D vector, 9 of which are features, and the 10th is the index label of morphology. The index labels of training records are known before training a model, while in test records, they are unknown waiting for prediction by the model. A training record can be shown as Table 3.3.

Table 3.3a training record

Table 3.3a training record
\begin{tabular}{|c|c|c|c|c|}
\hline $\mathbf{R}$ & F & H1 & H2 & H3 \\
\hline 0.513 & 44.6 & $5.45 \mathrm{E}-08$ & $1.47 \mathrm{E}-14$ & $2.17 \mathrm{E}-16$ \\
\hline H4 & H5 & H6 & H7 & \\
\hline $8.53 \mathrm{E}-17$ & $1.75 \mathrm{E}-32$ & $7.42 \mathrm{E}-24$ & $-1.47 \mathrm{E}-33$ & \\
\hline
\end{tabular}

\subsubsection{Training}

Kernel function is the core of SVM algorithm, by which SVM reaches the purpose of classification with little computation. In our experiment, we use RBF kernel function because experiment results indicate that it can reach an ideal classification effect without prior knowledge. RBF kernel function is like (3.1).

$$
\mathrm{K}\left(\mathrm{x}, \mathrm{x}_{\mathrm{i}}\right)=\exp \left(-\frac{\left|\mathrm{x}-\mathrm{x}_{\mathrm{i}}\right|^{2}}{\sigma^{2}}\right)(3.1)
$$

In such case, there are 2 arguments needed to be determined, one is penalty factor $\mathrm{C}$ and another is core parameter $\sigma$. The procedure of determining parameters is shown in Fig. 3.3. 


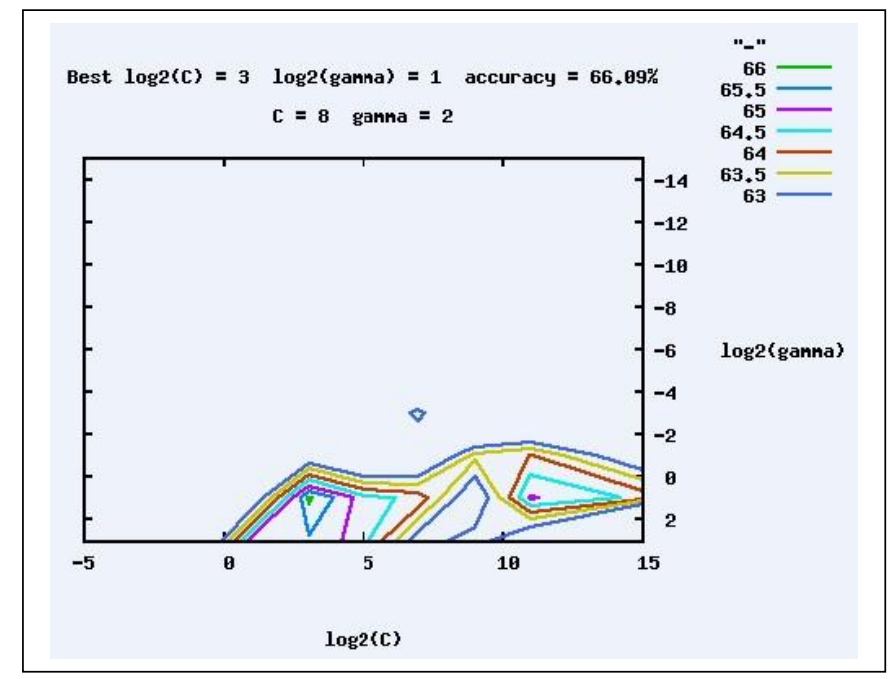

Fig. 3.3Parameter determination procedure

\subsection{Model Evaluation}

In order to verify the performance of the model, we need to use the test set prepared in advance. The prediction result is shown in Table 3.4.

Table 3.4Prediction result of test set

\begin{tabular}{lllllll}
\hline No. & sample & result & $\mathbf{0}$ & $\mathbf{1}$ & $\mathbf{2}$ & $\mathbf{3}$ \\
\hline $\mathbf{0}$ & 19 & 25 & 14 & 4 & 1 & 0 \\
$\mathbf{1}$ & 24 & 24 & 1 & 14 & 5 & 4 \\
$\mathbf{2}$ & 27 & 25 & 2 & 4 & 19 & 2 \\
$\mathbf{3}$ & 25 & 21 & 8 & 2 & 0 & 15 \\
\hline
\end{tabular}

According to Table 3.4, there are 95 records whose labels need to be predicted in total, in which 19 are standing, 24 are bending, 27 are crouching, and 25 are winging. While in the result, there are 62 records whose label are predicted correctly which means the accuracy of the model is $65.3 \%$.

In order to evaluate the model's performance of specific morphology, we define two parameters, one is precision $\sigma_{\mathrm{p}}$, and another is recall $\sigma_{\mathrm{r}}$, their definitions are as follows:

Suppose there are $\mathrm{m}$ records of morphology A (A can be one of standing, bending, crouching and winging), and in the prediction result there are $\mathrm{n} A$ records, in which $\mathrm{p}$ are correct, then $\sigma_{\mathrm{p}}=\frac{\mathrm{p}}{\mathrm{n}}$, and $\sigma_{\mathrm{r}}=\frac{\mathrm{p}}{\mathrm{m}}$.

The precision and recall of 4 morphologies are shown in Table 3.5. 


\begin{tabular}{cccccc}
\multicolumn{6}{c}{ Table 3.5Precision and recall of 4 morphologies } \\
\hline No. & sample & result & correct & precision & recall \\
\hline $\mathbf{0}$ & 19 & 25 & 14 & $56 \%$ & $73.7 \%$ \\
$\mathbf{1}$ & 24 & 24 & 14 & $58.3 \%$ & $58.3 \%$ \\
$\mathbf{2}$ & 27 & 25 & 19 & $76 \%$ & $70.4 \%$ \\
$\mathbf{3}$ & 25 & 21 & 15 & $71.4 \%$ & $60 \%$ \\
\hline
\end{tabular}

\section{Birds Behaviors Identification}

Bar-headed geese in Qinghai Lake are mainly for reproduction. Through longterm observation on breeding behavior of bar-headed geese, we summarized some basic behaviors of bar-headed geese in the breeding season: standing watch; walking; hatching; fighting. These behaviors can be identified by the basic postures mentioned in the previous section and finite time or location condition. Specific judgment conditions shown in Table 4.1:

Table 4.1 Decision conditions of basic behaviors

\begin{tabular}{|l|l|l|}
\hline Posture & Decisionconditions & Basic behavior \\
\hline Stand & the duration $t$ & standing watch \\
\hline Stand & $\begin{array}{l}\text { continuous change of lo- } \\
\text { cation }\end{array}$ & walking \\
\hline Crouch & the duration $t$ & hatching \\
\hline Bend & the duration $t$ & hatching \\
\hline Wing & the duration $t$ & fighting \\
\hline
\end{tabular}

Combining with the results of target detection, target tracking, posture classification and decision conditions of basic behaviors. We achieved a recognition system of bar-headed geese behaviors in monitor video. Through the validation using some 2012 monitor video, the recognition rate of 4 basic behaviors can reach about: standing watch $56 \%$; walking $56 \%$; hatching $64.7 \%$; fighting $71.4 \%$. Considering the complexity of the field real-time monitoring and the influence of many other factors, this rate is satisfying.

\section{Summary and scope}

We studied the classification of the basic postures of bar-headed geese, classified the four basic postures of bar-headed geese using SVM. Combined with target detection and target tracking technology, basic behavior decision condition of breeding season bar-headed geese, we realize the automatic identification of the four 
basic behavior of breeding season bar-headed geese. And recognition rate reached a certain height.

Next, we will continue to study the factors, which affect the recognition rate. Improve the basic behavior recognition rate. And research how to recognize abnormal behavior of breeding season bar-headed geese.

\section{References}

[1] SONGJian-hui, WEI Ning, HU Jian-bo, et al.. Setup of a video camera-computer monitoring system for study of sexual preference and sexual behavior of rats. Chinese Journal of Behavioral Medical Science. 2008, 15(10): 941-943.

[2] ZHANG Min. Study and Application of Animal Behavior Automatic Analysis Based on Posture Recognition. Zhejiang: Zhejiang University, Ph.D. Thesis. 2005.

[3] ZHANG Min, ZHANG Heng-yi, ZHENG Xiao-xiang. Automatic recognition of rat's postures based on contour curvature and hierarchical clustering analysis. Journal of Zhejiang University(Engineering Science). 2008, 40(3): 524-532.

[4] ZHANG Min, ZHANG Heng-yi, ZHENG Xiao-xiang. Automatic Recognition of Rat's Postures Based on Naive Bayes Classifier. Space Medicine \& Medical Engineering. 2005, 18(5) 370-374.

[5] L.Noldus, A. J.Spink and R.A. Tegelenbosch. Ethovision: A versatile video tracking system for automation of behavioral experiments. Behavior Research Methods, Instruments \& Computers, 2001, 33(3): 398-414.

[6] Med Associates Inc. Home Cage Video Tracking System. http://www.medassociates.com/new prod/video.htm, 2007, 11, 01.

[7] BIOBSERVE GmbH. Trackit system family. http://www.biobserve.com/products/trackit/index.htm, 2007, 11, 05.

[8] Tucher Balch, Zia Khan, Manuela Veloso. Automatically tracking and analyzing the behavior of live insect colonies. AGENTS'01, 2001: 521-528.

[9] OpenCV 2.4.3 documentation [EB/OL], [2012-12-26], http://docs.opencv.org. 